\title{
APLICACIÓN WEB PARA LA IDENTIFICACIÓN AUTOMÁTICA DEL USO DEL TAPABOCAS
}

\author{
WEB APPLICATION FOR AUTOMATIC \\ IDENTIFICATION OF THE USE OF MASKS
}

\section{Paula Andrea Rodríguez-Marín}

Doctora en Ingeniería de sistemas por la Universidad Nacional de Colombia (Medellín/Colombia)

Profesora en el Instituto Tecnológico Metropolitano de Medellín (Medellin/Colombia)

E-mail: Paularodriguez@itm.edu.co

\section{Victor Manuel Torres-Jiménez}

Técnologo en Electrónica por el Instituto Tecnológico Metropolitano (Medellín/Colombia)

Estudiante de Ingeniería Electrónica en el Instituto Tecnológico Metropolitano de Medellín (Medellin/Colombia)

E-mail: victortorres240693@correo.itm.edu.co

\section{Alejandro Piedrahíta-Carvajal}

Tecnólogo en Sistemas de información por el Instituto Tecnológico Metropolitano (Medellín/Colombia)

Estudiante de Ingeniería de Sistemas en el Instituto Tecnológico Metropolitano de Medellín (Medellin/Colombia)

E-mail: Alejandropiedrahita264000@correo.itm.edu.co

\section{Daniel Terraza-Arciniegas}

Tecnólogo en Electrónica por el Instituto Tecnológico Metropolitano (Medellín/Colombia)

Estudiante de Ingeniería Electrónica en el Instituto Tecnológico Metropolitano de Medellín (Medellin/Colombia)

E-mail: Danielterraza212285@correo.itm.edu.co

\section{Mauricio Amaya-Gómez}

Tecnólogo en Electrónica por el Instituto Tecnológico Metropolitano (Medellín/Colombia)

Estudiante de Ingeniería Electrónica en el Instituto Tecnológico Metropolitano de Medellín (Medellin/Colombia)

E-mail: Mauricioamaya189862@correo.itm.edu.co

\section{Leonardo Duque-Muñoz}

Doctor en Ingeniería por la Universidad de Antioquia (Medellín/Colombia)

Profesor en el Instituto Tecnológico Metropolitano de Medellín (Medellín/Colombia)

E-mail: Leonardoduque@itm.edu.co

\section{Juan David Martínez-Vargas}

Doctor en Ingeniería por la Universidad Nacional de Colombia (Manizales/Colombia) Profesor en el Instituto Tecnológico Metropolitano de Medellín (Medellín/Colombia)

E-mail: Juanmartinez@itm.edu.co 


\section{Brazilian

\section{RESUMEN}

Junto con el lavado de manos y el distanciamiento social, el uso de máscaras faciales (tapabocas) es una de las medidas de bioseguridad para prevenir la transmisión de enfermedades respiratorias como el covid-19. En este artículo proponemos la identificación automática del uso del tapabocas, con el objetivo de realizar alarmas del mal uso y el no uso del tapabocas. Este trabajo puede ser utilizado en lugares de acceso a personas donde el uso del tapabocas debe ser obligatorio. Se construyó una base de datos de imágenes con personas haciendo uso correcto del tapabocas (clase OK), mal uso del tapabocas ( clase BAD) y sin tapabocas (clase No-Mask). La base de datos fue construida por los estudiantes del semillero en inteligencia artificial del ITM. Para entrenar el modelo se implementó una red neural con arquitectura triplet, en la cual, después de normalizar cada imagen y transformarla en una de menor dimensión, se toma una imagen objetivo, llamado anchor, en este caso una de nuestras clases definidas, un ejemplo positivo (de la misma clase del anchor) y dos ejemplos negativos (de diferente clase del anchor, una por cada clase diferente). Con estas muestras se calcula la distancia entre el anchor y los ejemplos positivo y negativos. La distancia será grande entre el anchor y los ejemplos negativos y pequeña con el ejemplo positivo. Los ejemplos positivos y negativos se seleccionaron aleatoriamente. Los resultados son promisorios y se se hace una buena clasificación al llegar una muestra nueva.

Palabras clave: Tapabocas. Reconocimiento automático. Redes neuronales. Triplet.

\section{ABSTRACT}

Along with the handwash and the social distancing, the use of masks is one of the most common biosecurity measures to prevent the transmission of respiratory diseases as covid-19. In this paper, we propose a methodology for the automatic recognition of mask usages, to make bad and no use mask alerts. This work might be used in places where the use of masks is mandatory. Students of the ITM - Artificial Intelligence research group built an image database with three classes: correct, bad, and no-mask. To train the model, we implemented a triplet neural network that takes as inputs faces with normalized sizes and amplitudes. Then, an anchor face, a positive example (same class to the anchor), and two negative examples (each of a different class to the anchor) pass through the network where optimizing a triplet loss we seek for a new representation space where samples of same classes remain close and far from the remaining classes. Achieved results are promising as the system properly detects when someone is using properly a mask.

Keywords: Mask. Automatic recognition. Neural networks. Triplet. 


\section{Brazilian \\ Creative Industries}

\section{INTRODUCCIÓN}

Desde el año 2019, todos los países del mundo se vieron ampliamente afectados por la llegada del nuevo coronavirus (SARS-CoV-2; el agente causante del COVID-19), que se propaga a través del aire cuando una persona que está infectada suelta gotas de saliva, al toser o estornudar, inclusive cuando se habla o se levanta la voz, y otra persona está cerca y estas gotas se diseminan o ingresan por la nariz o boca de la persona o personas sanas. Los síntomas más comunes son la falta de aire, tos, dolor, fiebre, somnolencia (HASAN et al., 2020).

La llegada de la pandemia trajo retos en todas las áreas del conocimiento y al no tener un efectivo medicamento de laboratorio para aliviar esta dolencia, se han ampliado la oferta de elementos de cuidado personal, para evitar el contagio por el covid19, esto activó el desarrollo de nuevos dispositivos y materiales para cubrir los requerimientos de bioseguridad y protección de las vías aéreas para la comunidad (BRICEÑO et al., 2020). Es así como el lavado de manos, el uso de máscaras faciales (tapabocas) y el mantenimiento de la distancia social se han identificado como las medidas más eficaces contra COVID-19 y se recomiendan como la "nueva normalidad" (HASAN et al., 2020), afirmando que el uso generalizado de tapabocas podría ayudar a reducir aún más el riesgo de transmisión del virus (JIMÉNEZ MORA et al., 2020).

Hay investigaciones que llegan a afirmar que el uso de mascarillas / tapabocas en lugares públicos son muestra de solidaridad social y es la respuesta a la pandemia mundial (CHENG; LAM; LEUNG, 2020), sin embargo, monitorear manualmente si una persona si está llevando el tapabocas de forma correcta y realizar una alarma, no es una tarea fácil, por lo tanto se deben buscar enfoques más eficientes basados en el área de inteligencia artificial de visión artificial o visión por computador, que monitoree las personas en tiempo real cuando pasen por un lugar público y se señale si la persona lleva bien puesto el tapabocas, mal puesto o simplemente no lleva el tapabocas (SURESH; PALANGAPPA; BHUVAN, 2021)

En la actualidad, existen varias modelos para realizar el reconocimiento automático del uso del tapabocas (NAGRATH et al., 2021) y se está experimentando avances en el procesamiento de imágenes y visión por computador, para solucionar este problema, y llegar a realizar alertas sobre el uso inadecuado de este elemento protector.

Por otro lado, el aprendizaje profundo ha demostrado un enorme potencial en muchas aplicaciones de la vida real en diferentes dominios. Uno de estos potenciales es la detección de objetos, donde se han logrado resultados prometedores en relación con el hallazgo de un objeto dentro de una imagen (LOEY et al., 2021).

En este artículo proponemos un modelo de detección automática del tapabocas, que alerte a las personas de su uso adecuado, que puede ser implementado en un lugar público, enfocándonos solo 


\section{Brazilian

en la conceptualización e implementación del algoritmo, para ello se seleccionó una red neuronal con arquitectura triplet, donde la entrada es un conjunto de imágenes etiquetadas.

El documento esta organizado como sigue, primero se da una conceptualización teórica, seguido por algunos trabajos relacionados, en la sección 4 se presenta la metodología propuesta para el modelo de detección automática del uso del tapabocas, en la sección 5 los resultados encontrados al implementar el modelo en una aplicación web y finalmente las consideraciones finales.

\section{FUNDAMENTACIÓN TEÓRICA}

En esta sección se abordarán los principales conceptos en los que se enmarca esta investigación, como lo son el covid-19, el uso del tapabocas y las redes neuronales.

\subsection{COVID-19}

El virus del síndrome respiratorio agudo grave coronavirus 2 (SARS-CoV-2), es responsable de la enfermedad por coronavirus 2019 (JIMÉNEZ MORA et al., 2020), es una enfermedad infecciosa que afecta de distintas maneras en función de cada persona. La mayoría de las personas que se contagian presentan síntomas de intensidad leve o moderada, y se recuperan sin necesidad de hospitalización.

El virus que causa la COVID-19 se transmite principalmente a través de las gotas de saliva generadas cuando una persona infectada tose, estornuda o espira. Estas pequeñas gotas son demasiado pesadas para permanecer suspendidas en el aire y caen rápidamente sobre el suelo o las superficies. Una persona puede infectarse al inhalar el virus si está cerca de una persona con COVID-19 o si, tras tocar una superficie contaminada, se toca los ojos, la nariz o la boca.

\subsection{TAPABOCAS}

También llamado mascarilla, máscara facial o cubre bocas, el tapabocas es un descubrimiento chino del sinólogo Joseph Needham. Desde finales del siglo XIX se recomienda su uso para la prevención, de personal médico, contra infecciones contagiosas, pero con la llegada de la gripe española a principios del siglo XX, los tapabocas fueron imprescindibles para todas las personas como la mejor herramienta para combatir el virus.

En 2003 el uso de tapabocas alcanzó se popularizó debido al SARS (síndrome respiratorio agudo) esparcido en China, así mismo en 2004 con la llegada de la gripe aviar y en 2009 con la gripe AH1N1 los tapabocas fueron los protagonistas para prevenir el contagio a miles de personas (BRICEÑO et al., 2020). 


\section{Brazilian \\ Creative Industries}

En la actual pandemia por COVID-19, el correcto uso de tapabocas ayuda al no contagio y es ordenado y obligado su uso por los gobiernos de diferentes países.

\subsection{REDES NEURONALES}

Las redes neuronales son un modelo computacional que se esta popularizando para la solución de diferentes tareas, consta de un conjunto de unidades llamadas neuronas artificiales conectadas entre sí a través de enlaces. En estos enlaces la salida de la neurona se multiplica por un peso, el cual puede aumentar o disminuir, así mismo como activar o desactivar neuronas vecinas, su objetivo es cumplir con la función de activación. Dentro de las redes neuronales, se encuentran la red neuronal de convolución (CNN), que es una clase de red neuronal profunda (DNN), (SURESH; PALANGAPPA; BHUVAN, 2021), comúnmente utilizada en la clasificación y el reconocimiento de imágenes.

Una arquitectura de red de las CNN es el método triplet, usado en este artículo, y que fue propuesto para el reconocimientos de rostros en imágenes. Triplet optimiza el espacio de búsqueda de las entidades del mismo tipo que se mantienen cerca y de diferente clase que se mantienen alejadas. Para aplicar esta arquitectura es necesario comparar parede de imágenes de ejemplos positivos (misma clase) y ejemplos negativos (diferentes clases).

\section{TRABAJOS RELACIONADOS}

A continuación, se presentan algunos trabajos relacionados, con el reconocimiento automático del tapabocas.

En (NAGRATH et al., 2021), proponen un modelo hibrido, combinando el aprendizaje automático clásico con el aprendizaje profundo para la detección de máscaras faciales (tapabocas), para la extracción de características utilizan Resnet50 y para la clasificación utilizan árboles de decisión, Support Vector Machine (SVM) y algoritmo de conjunto. La arquitectura utilizada, consta de OpenCV Deep Neural Network (DNN), TensorFlow, Keras y MobileNetV2, que se utiliza como clasificador de imágenes. Concluyen que el uso de OpenCV DNN con ResNet-10 como columna vertebral, es capaz de detectar rostros en la mayoría de las orientaciones. Y que el modelo propuesto entrega predicciones ligeras pero precisas para la clasificación en función de si se usa un tapabocas o no se usa. Así mismo el modelo propuesto por (LOEY et al., 2021) tiene dos componentes, el primero es para la extracción de características, también usando el modelo de aprendizaje de transferencia profunda ResNet-50 y el segundo para la detección de mascarillas médicas basadas en YOLO v2. 


\section{Brazilian \\ Creative Industries}

Por otra parte, en el trabajo de (SURESH; PALANGAPPA; BHUVAN, 2021), proponen el monitoreo en tiempo real a las personas en público, para clasificarlas en si usan o no un tapabocas, el modelo en tiempo real utiliza una red neuronal de convolución para el reconocimiento de imágenes junto con los algoritmos de TensorFlow y Keras para realizar la detección si una persona está usando un tapabocas. Realizaron el entrenamiento con los datos de Kaggle y utilizaron MobileNet para el entrenamiento y clasificación de imágenes de alta calidad. El sistema se ejecuta en tiempo real y detecta si un rostro individual tiene máscara facial, si no, notifica al individuo personalmente a través de un mensaje de texto.

\section{METOdOLOGÍA}

En esta sección se explica el desarrollo del modelo propuesto para el reconocimiento automático del uso correcto del tapabocas.

\subsection{ADQUISICIÓN DE LA BASE DE DATOS}

Para realizar el proceso de entrenamiento, primero se adquirió una base de datos propia, donde se le pidió a los estudiantes del semillero de inteligencia artificial adscrito al grupo de investigación de máquinas inteligente y reconocimiento de patrones (MIRP), del Instituto Tecnológico Metropolitano de Medellín - Colombia, que se tomaran fotos ellos y sus familias con las tres clases que se quieren clasificar, es decir tapabocas bien puesto, tapabocas mal puesto y sin tapabocas, con el objetivo de entrenar el modelo propuesto.

Después de la primera solicitud de imágenes se descubrió que la iluminación era importante al igual que la inclinación y dirección del rostro. En un sistema ideal los rostros deben estar con la iluminación adecuada y el rostro frontal, pero también se debe considerar cuando el rostro de la persona esta en otras posiciones, por ejemplo otros ángulos y con mala iluminación. Por esto se realizó entonces una segunda solicitud de fotos, donde no todos los rostros estuvieran de frente y con buena iluminación y existieran otros elementos de fondo.

Con las dos solicitudes de fotos, para la clase tapabocas mal puesto se recopilaron 196 imágenes, para el tapabocas bien puesto 98 y finalmente para la clase sin uso del tapabocas se contó con 73 imágenes.

En la figura 1 se presenta parte de la base de datos adquirida con las dos solicitudes de fotos y las tres clases. 


\section{Brazilian \\ Creative Industries}

Figura 1. Parte de la base de datos adquirida.

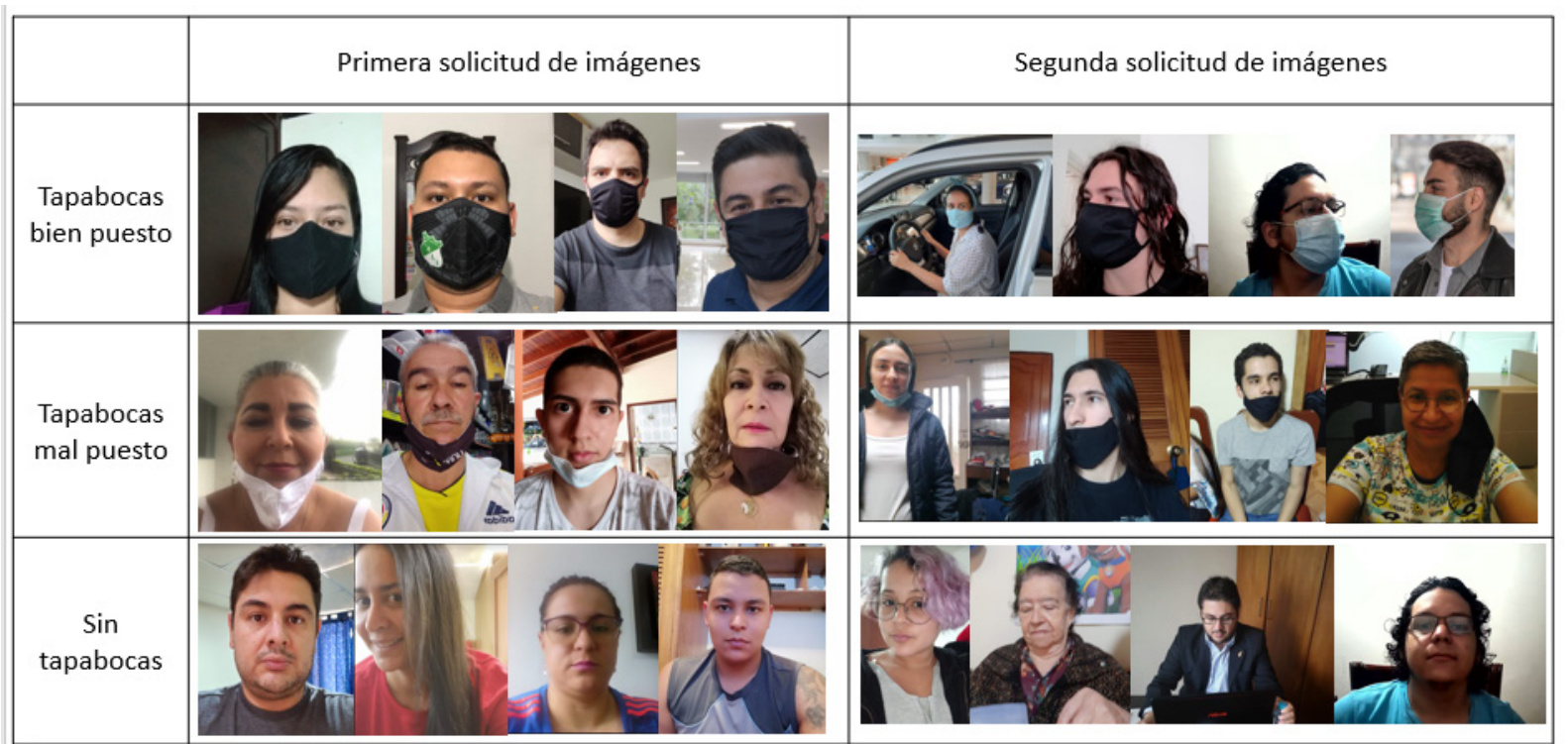

Fuente propia.

\subsection{RECONOCIMIENTO DEL ROSTRO}

Para solucionar la tarea si una persona tiene bien puesto su tapabocas, es necesario primero reconocer el rostro de las personas, ubicar sus contornos, para finalmente ubicar si el tapabocas esta bien puesto, mal puesto o si la persona no tiene tapabocas. Se analizaron varias propuestas o metodologías para el reconocimiento de rostros utilizando visión artificial como (ANGGO; LA ARAPU, 2018), (SHEN; KHANNA, 1997), (MOHAMMED et al., 2018), (WILLIAM et al., 2019) (WINARNO et al., 2019), (LI et al., 2017), (VIOLA; JONES, 2001), y se seleccionó el modelo de red neuronal convolucional multitarea MTCNN (Multitask Cascaded Convolutional Networks), descrita en (LI et al., 2018) (ZHANG et al., 2016), cuya metodología propone la obtensión de 5 puntos fiduciales del rostro de la persona, además de los puntos que enmarcan el rostro de una persona en una imagen, llamados bouding boxes, con el punto superior izquierdo e inferior derecho. Para nuestro trabajo utilizamos solo los bounding boxes, debido que los puntos fiduciales no aparecen en dos de las clases propuestas.

\subsection{ENTRENAMIENTO Y PRUEBA DEL MODELO}

Para entrenar nuestro modelo de reconocimiento de tapabocas, utilizamos una red neuronal con arquitectura triplet como se ve en la figura 2. Esta red se entrena con los rostros obtenidos después de pasar cada cuadro de video por el modelo MTCNN, i.e., cada cuadro de video se representa como un tensor 


\section{Brazilian Creative Industries}

de dimensión (Nf×3×160×160) donde Nf indica el número de rostros en el cuadro, 3 indica el número de canales (RGB) y 160x160 es el número de píxeles en cada canal que conforma cada rostro.

Figura 2. Arquitectura de la etapa de entrenamiento

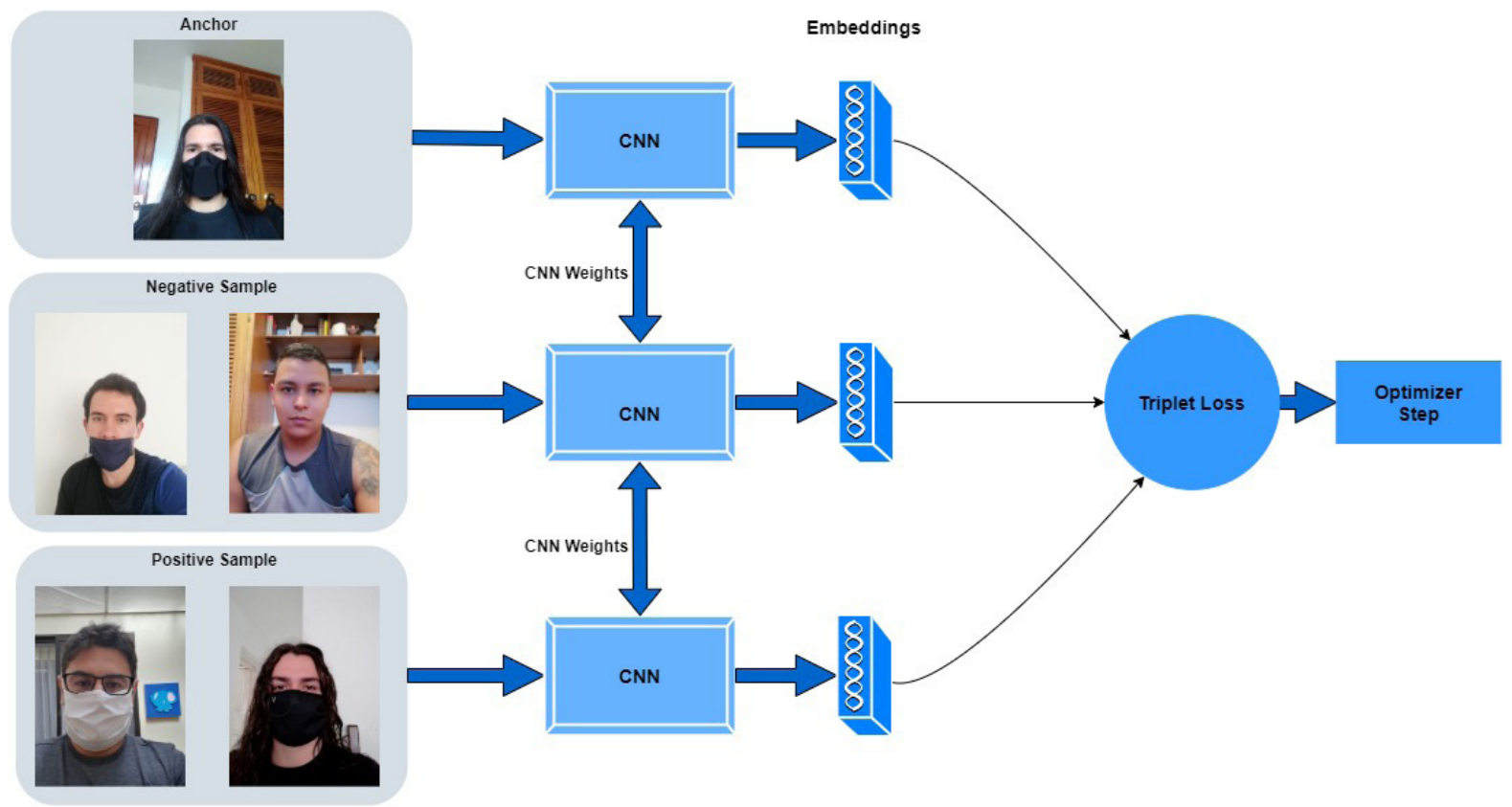

Para el entrenamiento, la arquitectura triplet consta de dos bloques principales: un codificador (encoding) y una función de pérdida triplet. El codificador se encarga de transformar los rostros de entrada a un conjunto de puntos de menor dimensión. En este caso, para fines de visualización, los 160x160 pixeles se transformaron a 3 puntos utilizando dos capas convolucionales con maxpooling y activaciones ReLU y dos capas Fully Connected con dropout para evitar el sobrenetrenamiento y funciones de activación ReLU.

La función de costo triplet toma un ejemplo objetivo (anchor), un ejemplo positivo (de la misma clase del anchor) y un ejemplo negativo (de diferente clase del anchor). Con estos ejemplos se calcula un valor que va a ser grande si en el nuevo espacio de representación la distancia entre el anchor y el ejemplo negativo es más pequeña que la distancia entre el anchor y el ejemplo positivo; y va a ser pequeño en caso contrario. De esta forma, minimizando esta función de costo con respecto a los parámetros de la red neuronal, se espera que en el nuevo espacio de representación los ejemplos pertenecientes a la misma clase se agrupen lejos de los ejemplos pertenecientes a las demás clases. Dado que en nuestra experimentación teníamos 3 clases, en el entrenamiento cada ejemplo de cada clase se pasó por la red neuronal con 2 ejemplos negativos, 1 de cada clase. Los ejemplos positivos y negativos se seleccionaron aleatoriamente. Los modelos se entrenaron dividiendo los datos en $80 \%$ para entrenamiento y $20 \%$ para 


\section{Brazilian \\ Creative Industries}

prueba. De los datos de entrenamiento, se deja un $20 \%$ adicional para validación con el fin de sintonizar los parámetros del modelo. Un ejemplo del espacio de representación obtenido se puede ver al final de la primera fila de la figura 3.

Figura 3. Arquitectura para la predicción

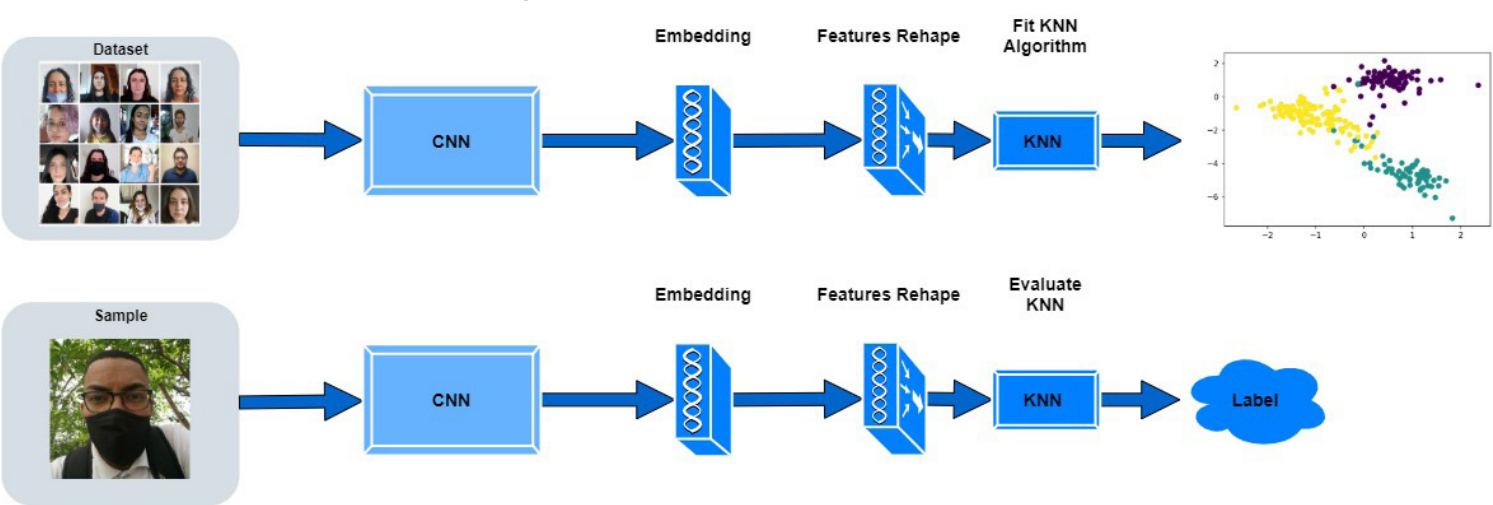

Para la parte de validación y prueba, se entrenó un clasificador de k vecinos más cercanos (kNN) sobre el espacio de representación resultante. De esta forma, cuando llega un rostro sin etiqueta, se pasa a través del encoder y se le asigna la etiqueta de los 3 vecinos más cercanos, como se puede ver en la figura 3.

\subsection{APLICATIVO WEB PARA EL USO DEL MODELO}

Para el uso del modelo propuesto, se implementó un proyecto en FLASK descrito en (ASLAM et al., 2015), como una aplicación web que permitiera el uso de los algoritmos. Para esto se siguió la arquitectura de programación modelo - vista - controlador, explicada en (MUFID et al., 2019)", que facilita la implementación de aplicativos webs organizando por capas de trabajo y diferenciando las funciones de cada una de ellas. En la capa de vista se programan en HTML la interacción con el usuario final, además se utilizó CSS para dar estética y javascript para dar funcionalidad. En la capa de modelo, se implementa el modelo del reconocimiento automático del uso de tapabocas. La capa de controlador es la encargada de relacionar el modelo y la vista, para ello se utiliza FLASK. La selección de este microframework se dio por su estructura sencilla y su baja curva de aprendizaje, además por su optimización en las tareas sencillas.

Adicionalmente, se utilizó Visual Studio Code, un entorno de desarrollo integrado por su peso ligero y su fácil instalación de complementos y lenguajes de programación. Además, también integra un panel de control para facilitar la ejecución de la aplicación.

Para el acceso a la cámara del computador del usuario se creó el archivo, dentro de la capa del controlador, llamado camera.py, que se encarga de prender la cámara y extraer la información frame a 


\section{Brazilian \\ Creative Industries}

frame. Se creo tapabocas.py, que ejecuta el modelo entrenado, obtiene los frames de la cámara para procesarlos y devolver una predicción pertinente con alguna de las 4 clases seleccionada. En la capa del modelo se almacenan los XML y los códigos de la arquitectura de la red neuronal.

\section{RESULTADOS Y DISCUSIÓN}

En esta sección se presenta el funcionamiento de la aplicación web desarrollada y la integración con el modelo propuesto para la detección automática del uso del tapabocas.

El modelo clasifica una imagen, primero identificando el rostro en la imagen que es señalada con un cuadro, después la imagen pasa por el modelo y se genera una etiqueta dependiendo de lo encontrado en el modelo entrenado. Las etiquetas son:

1. Tapabocas bien puesto: $\mathrm{OK}$

2. Tapabocas mal puesto: BAD

3. Sin tapabocas: NO MASK

Se realizó la etiqueta en inglés para que fuera internacional y se pudiera utilizar en cualquier lugar del mundo.

Al ingresar a la aplicación el usuario se encuentra con la pantalla presentada en la figura 4, donde se le da la bienvenida a la aplicación y hay un menú a la izquierda, donde se puede navegar por las diferentes opciones.

\section{Figura 4 Vista de la página inicial de la aplicación web}

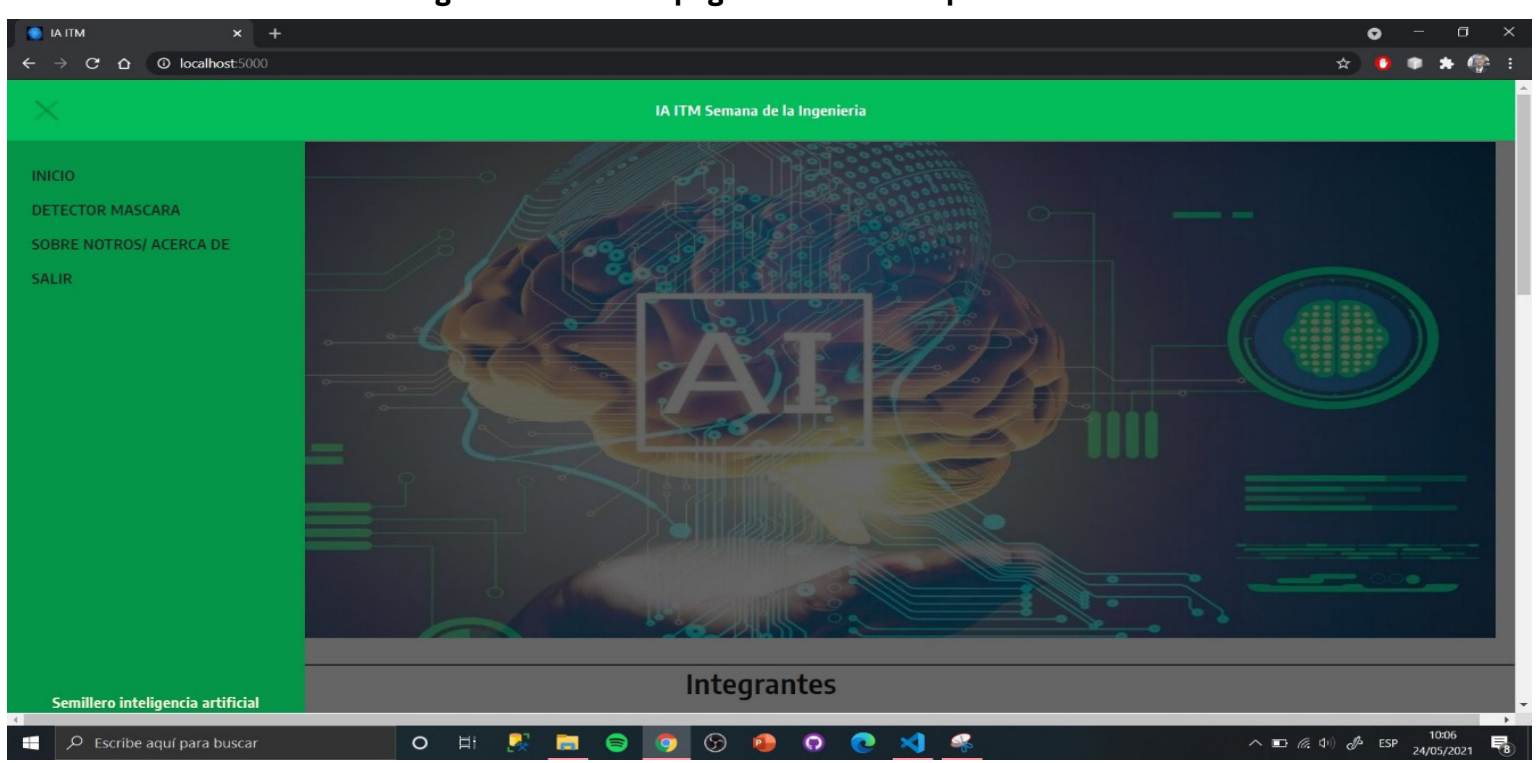




\section{Brazilian Creative Industries}

En la sección sobre nosotros, se presenta un vídeo del funcionamiento del sistema Figura 5 a y se da una explicación del modelo Figura 5 b y de la base de datos utilizada en la detección del tapabocas, figura $5 c$.

Figura 5. Vista de la sección sobre nosotros

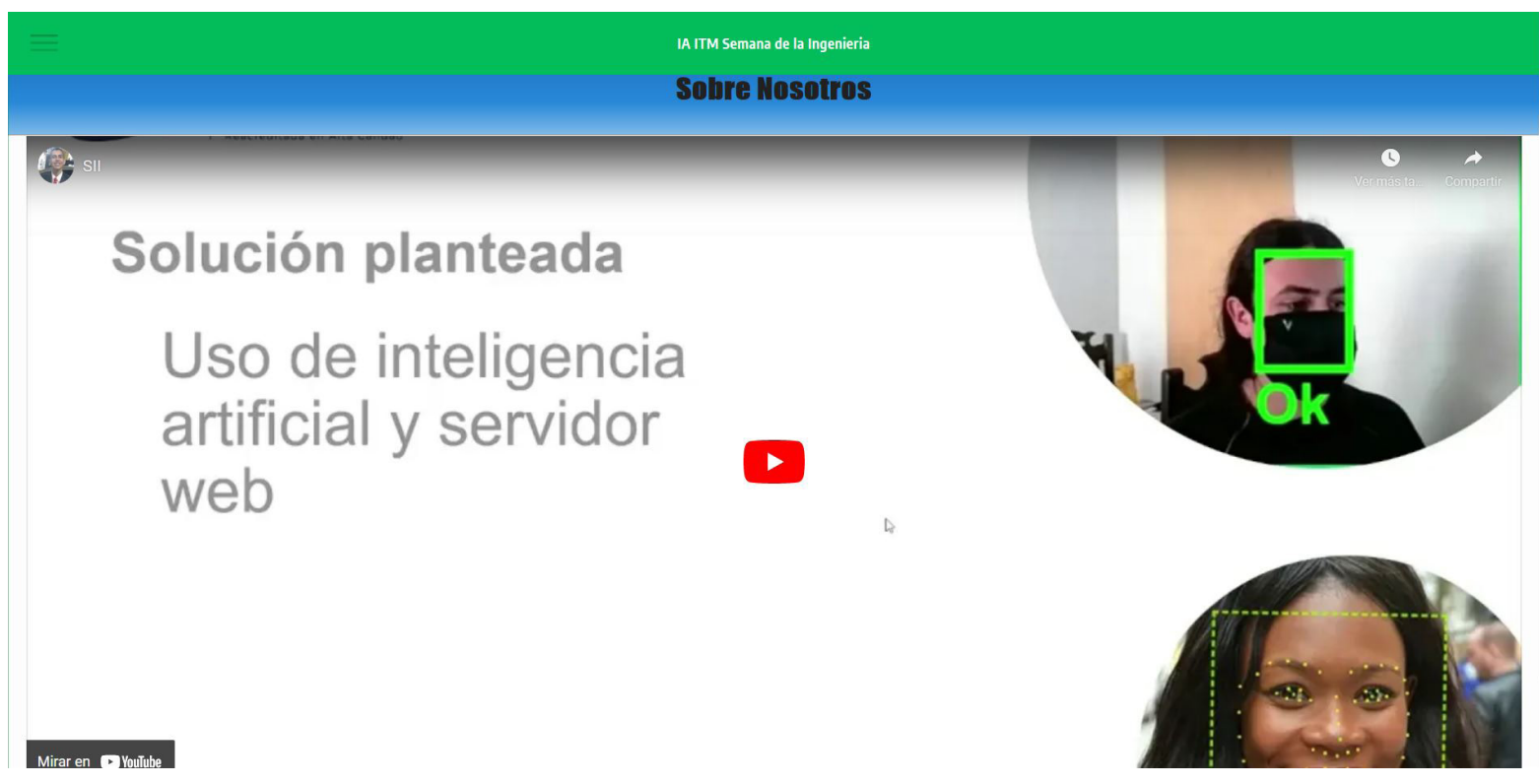

(a)

\section{INTRODUCCION}

La inteligencia artificial (IA) es la inteligencia llevada a cabo por máquinas. En ciencias de la computación, una máquina inteligente es un agente flexible que percibe su entorno y lleva a cognitivas que los humanos asocian con otras mentes humanas, como por ejemplo: percibir, razonar, aprender $y$ resolver problemas aplica cuando una máquina imita las funciones

Si se profundiza mas en la inteligencia artificial es de notar la existencia de una rama que, en los ultimos años, a tomado fuerza entre los apasionados por la creacion de maquinas inteligentes y es la Vision Artificial. Es definida como un campo de la inteligencia artificial que busca (mediante tecricas adecuadas) la obtencion, procesamiento y analisis de informacio

La premisa de este proyecto nace a partir de la dificil situacion mundial que se vive actualmente por un virus, poniendo en alerta a todos e infundiendo de manera tajante un cambio en Is vida cotidiana de las personas. Despues de meses de encierro en cuarentena, Colombia ha decidido reestablecer el comercio para varios establecimientos (si no es que ha todos) con algunas restricciones y normas que deben tener dichos establecimientos para permitir el ingreso de las pers
distanciamiento de 1.5 a 2 metros, entre otras que son mas especificas para cada tipo de negocio.

Aqui, se implementa un modelo de red neuronal capaz de (vision artificial) reconocer que personas cumplen o no con la restriccion de llevar tapabocas en espacios publicos y establecimientos comerciales, ademas, de indicar en que me dida se cumple con esta restriccion o no.

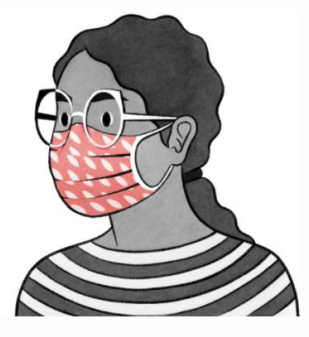

(b) 


\section{Brazilian Creative Industries}
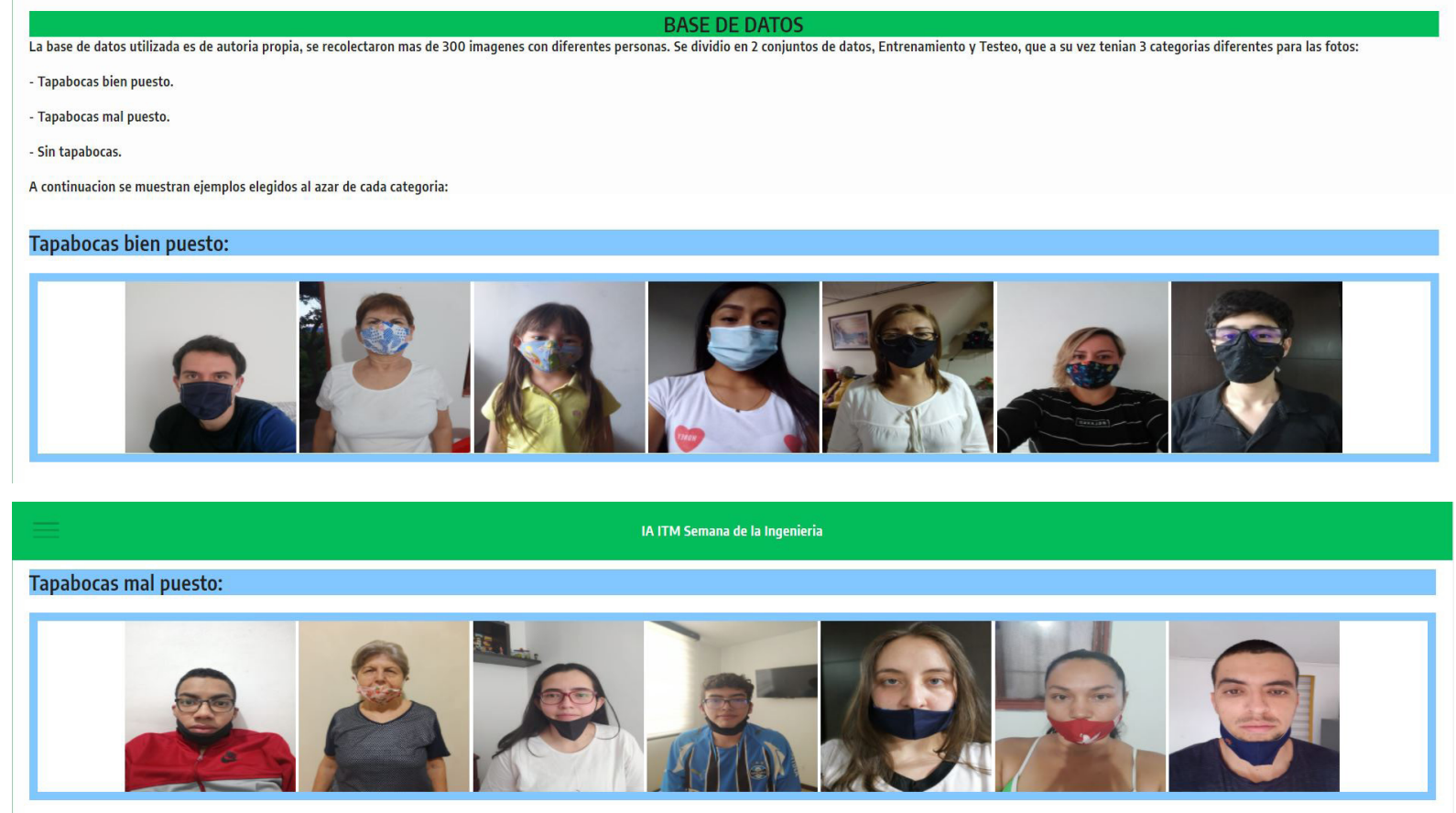

Sin tapabocas:

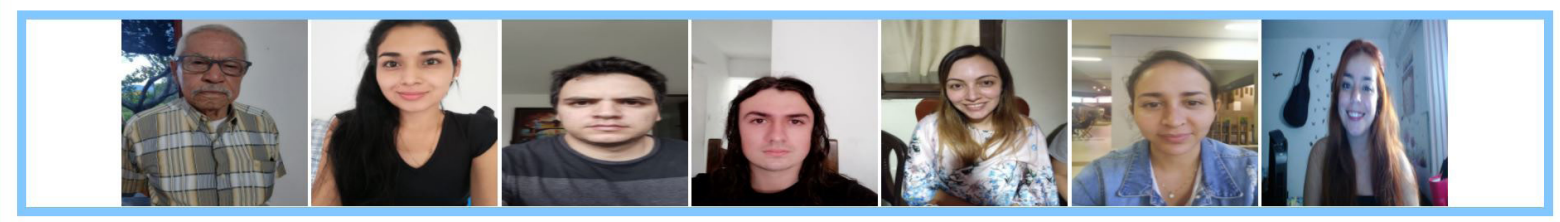

(c)

Finalmente, se presenta el funcionamiento del sistema, en la sección "Detector Máscara", donde se activa la cámara del equipo de cómputo donde se esté ejecutando la aplicación web. En las figuras 6 desde la a hasta la d, se presenta el resultado de la ejecución del detector automático de tapabocas implementado. 


\section{Brazilian Creative Industries}

Figura 6. Vista del funcionamiento en tiempo real del modelo implementado.

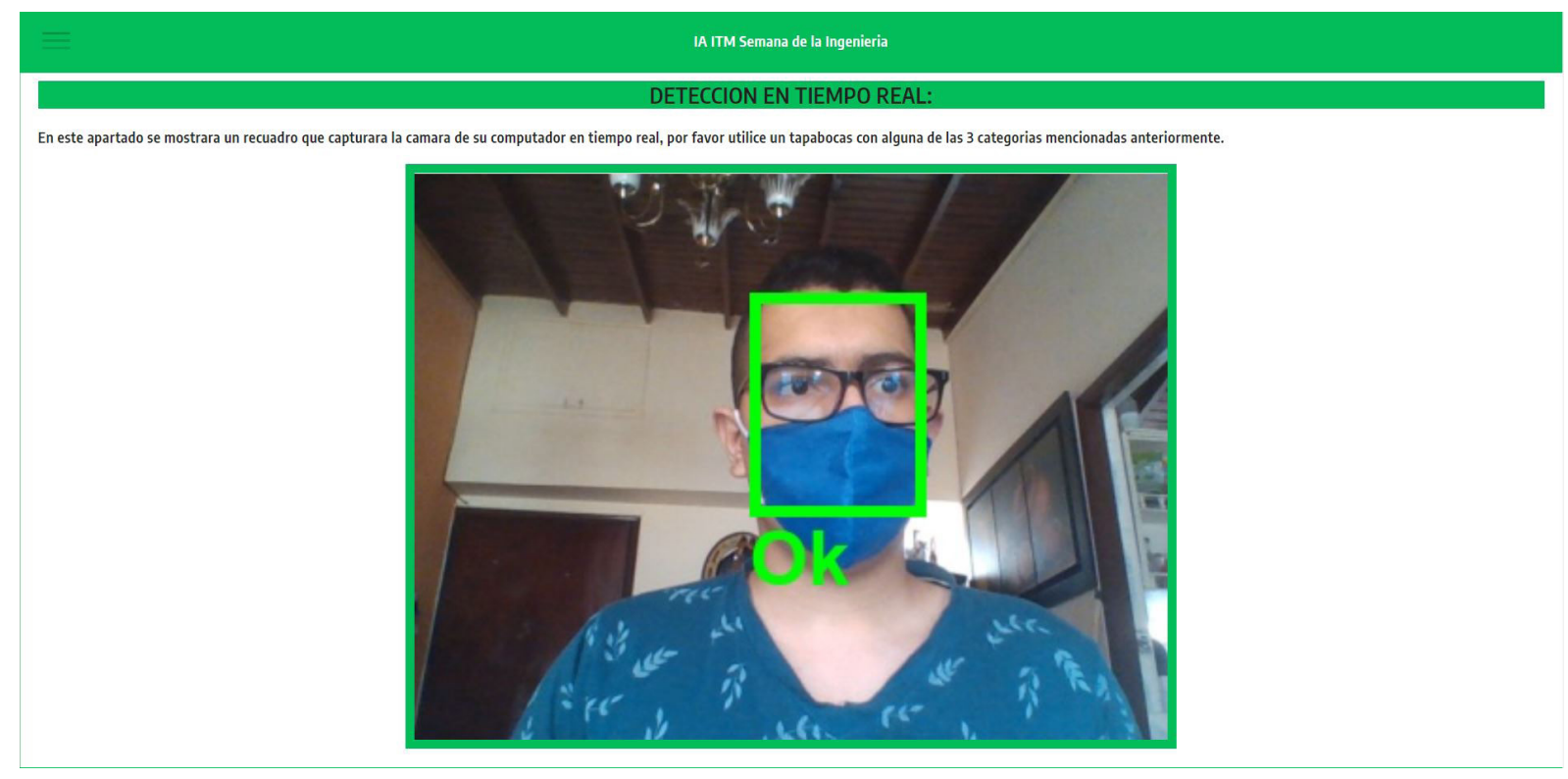

(a)

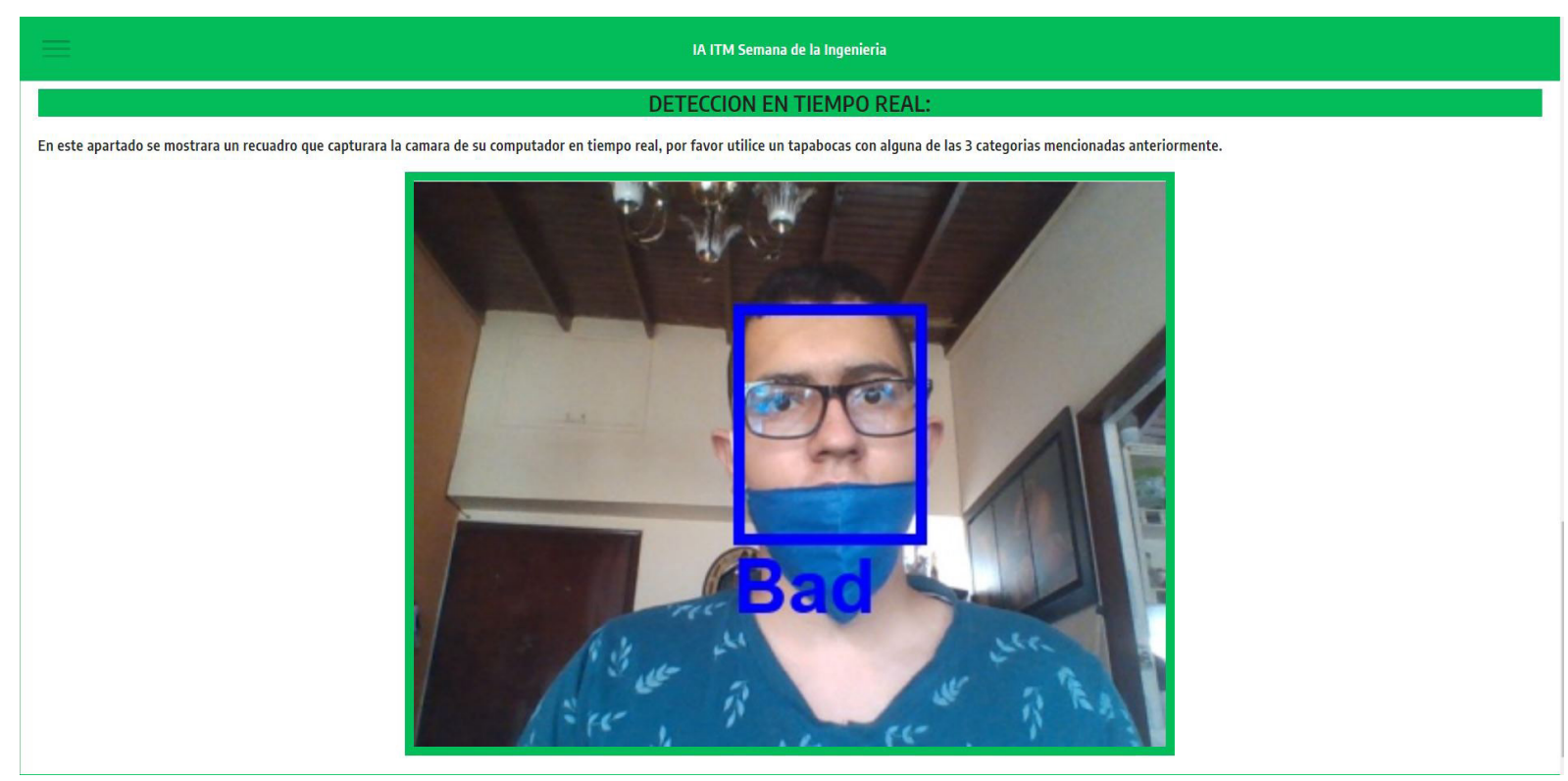

(b) 


\section{Brazilian Creative Industries}

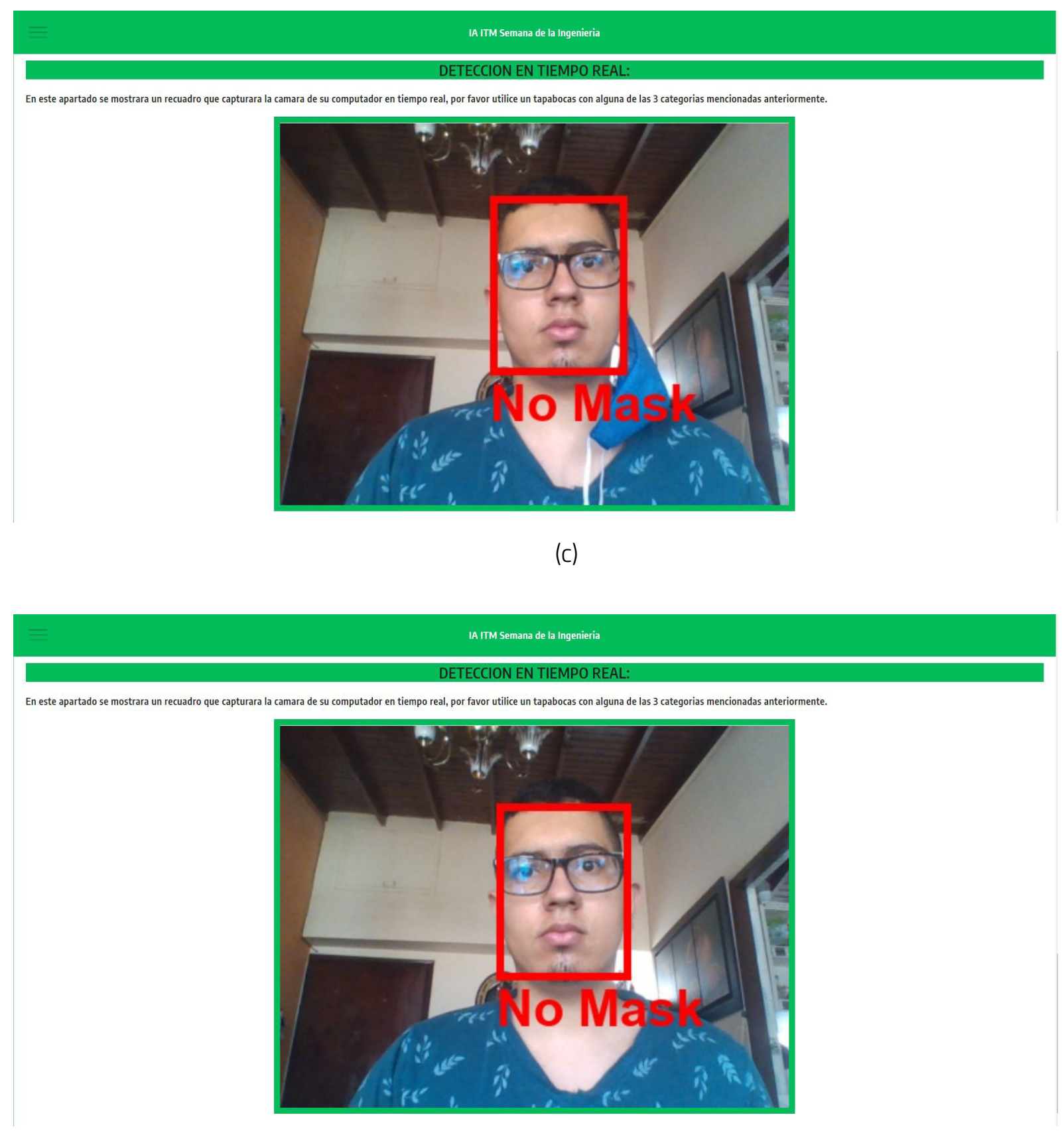

(d) 


\section{Brazilian \\ Creative Industries}

Para finalizar, se ejecutó la aplicación en el navegador Google Chrome y no se presentan errores de visualización ni de lentitud al ejecutar y navegar por las diferentes secciones. Se tomó el tiempo de carga para visualizar la aplicación dando 9.47 segundos en promedio.

\section{CONSIDERACIONES FINALES}

El objetivo de usar tapabocas es reducir la tasa de transmisión y propagación, dado que funciona como una barrera entre las mucosas y el medio ambiente. La recomendación de la Organización Mundial de la Salud es utilizar siempre el tapabocas, en especial en lugares cerrados con muchas personas. El uso del tapabocas previene el contagio de enfermedades respiratorias cuando hay contacto directo entre una persona infectada y una sana. En la actualidad por la pandemia generada por el COVID-19. Siempre debe recomendarse el uso del tapabocas con el lavado de manos y el distanciamiento social como medidas de bioseguridad y protección.

En este artículo se propone un modelo para la detección automática del uso de tapabocas o mascarilla, que ayuda a realizar un monitoreo de que personas están usando bien, mal o no usan esta herramienta, para así informar y tomar algunas medidas más estrictas y ayudar a la no propagación del covid-19. El modelo puede implementarse en cámaras de vigilancia en escuelas, universidades, centros comerciales, entre otros lugares públicos, donde se hace indispensable cubrir nariz y boca adecuadamente. Con este modelo se ayudará a romper la cadena de contagio cuando una persona este cerca de otra.

Para frenar la propagación del covid-19, usar tapabocas es una medida conveniente y efectiva.

\section{AGRADECIMIENTOS}

Este trabajo se desarrolló bajo el proyecto de investigación "Fortalecimiento y consolidación del Grupo de Investigación Máquinas Inteligentes y Reconocimiento de Patrones (MIRP), para responder a las necesidades de las industrias 4.0", con código P21103 financiado por la convocatoria de fortalecimiento de grupos investigación ITM para responder a las necesidades de la cuarta revolución industrial 2020. Además, apoyado por el semillero de inteligencia artificial, sus miembros y familiares quienes aportaron fotos para el entrenamiento y validación del modelo. 


\section{Brazilian \\ Creative Industries}

\section{REFERENCIAS}

ANGGO, M.; LA ARAPU. Face Recognition Using Fisherface Method. Journal of Physics: Conference Series, v. 1028, n. 1, p. 998-1001, 2018.

ASLAM, F. A. et al. Efficient Way Of Web Development Using Python And Flask. International Journal of Advanced Research in ComputerScience, v. 6, n. 2, p. 54-57, 2015.

BRICEÑO, J. C. et al. El boom de los tapabocas. Revista de ingeniería, v. 50, p. 72-75, 2020.

CHENG, K. K.; LAM, T. H.; LEUNG, C. C. Wearing face masks in the community during the COVID-19 pandemic: altruism and solidarity. The Lancet, v. 2019, n. 20, p. 2019-2020, 2020.

HASAN, N. A. et al. Face Masks - Protecting the Wearer But Neglecting the Aquatic Environment? SSRN Electronic Journal, v. 4, n. April, p. 100126, 2020.

JIMÉNEZZ MORA, M. A. et al. Uso de Tapabocas en la Población General para Controlar la Transmisión de COVID-19 : ¿ Cuál es la Evidencia ? Facultad medicina, p. 1-10, 2020.

LI, C. et al. Human face detection algorithm via Haar cascade classifier combined with three additional classifiers. ICEMI 2017 - Proceedings of IEEE 13th International Conference on Electronic Measurement and Instruments, v. 2018- Janua, p. 483-487, 2017.

$\mathrm{LI}$, J. et al. Robust face recognition using the deep C2D-CNN model based on decision-level fusion. Sensors (Switzerland), v. 18, n. 7, p. 1-27, 2018.

LOEY, M. et al. Fighting against COVID-19: A novel deep learning model based on YOLO-v2 with ResNet-50 for medical face mask detection. Sustainable Cities and Society, v. 65, October 2020, p. 102600, 2021.

MOHAMMED, N. N. et al. Face Recognition Based on PCA with Weighted and Normalized Mahalanobis distance. International Conference on Intelligent Informatics and Biomedical Sciences (ICIIBMS). Anais...IEEE, 2018

MUFID, M. R. et al. Design an MVC Model using Python for Flask Framework Development. IES 2019 - International Electronics Symposium: The Role of Techno-Intelligence in Creating an Open Energy System Towards Energy Democracy, Proceedings, n. Mvc, p. 214-219, 2019. 


\section{Brazilian Creative Industries}

NAGRATH, P. et al. SSDMNV2: A real time DNN-based face mask detection system using single shot multibox detector and MobileNetV2. Sustainable Cities and Society, v. 66, n. December 2020, p. 102692, 2021.

SHEN, W.; KHANNA, R. Prolog to Face Recognition: Eigenface, Elastic Matching, and Neural Nets. Proceedings of the IEEE, v. 85, n. 9, p. 1422, 1997.

SURESH, K.; PALANGAPPA, M. B.; BHUVAN, S. Face Mask Detection by using Optimistic Convolutional Neural Network. Proceedings of the 6th International Conference on Inventive Computation Technologies, ICICT 2021, p. 1084-1089, 2021.

VIOLA, P.; JONES, M. Rapid object detection using a boosted cascade of simple features. Proceedings of the IEEE Computer Society Conference on Computer Vision and Pattern Recognition, v. 1, p. 11-18, 2001.

WILLIAM, I. et al. Face Recognition using FaceNet (Survey, Performance Test, and Comparison).

Proceedings of $\mathbf{2 0 1 9}$ 4th International Conference on Informatics and Computing, ICIC 2019, 2019.

WINARNO, E. et al. Attendance System Based on Face Recognition System Using CNN-PCA Method and Real-Time Camera. 2019 2nd International Seminar on Research of Information Technology and Intelligent Systems, ISRITI 2019, p. 301-304, 2019.

ZHANG, K. et al. Joint Face Detection and Alignment Using Multitask Cascaded Convolutional Networks. IEEE Signal Processing Letters, v. 23, n. 10, p. 1499-1503, 2016. 